\title{
The Philosophical Significance of Tennenbaum's Theorem
}

\author{
Tim Button $8 \mathcal{F}$ Peter Smith
}

Notice. This paper is due to appear in Philosophia Mathematica. This paper may be subject to minor changes. The authoritative version should be obtained from Philosophia Mathematica.

Tennenbaum's Theorem yields an elegant characterisation of the standard model of arithmetic. Several authors have recently claimed that this result has important philosophical consequences: in particular, that it offers us a way of responding to model-theoretic worries about how we manage to grasp the standard model. We disagree. If there ever was such a problem about how we come to grasp the standard model, then Tennenbaum's Theorem doesn't help.

\section{Appealing to Tennenbaum's Theorem}

Tennenbaum's Theorem tells us that the only model of PA (first-order Peano Arithmetic) whose interpretation of addition and/or multiplication is recursive is the standard model.

To explain a bit further. It is (relatively) standard practice to distinguish models of a theory only up to isomorphism. Adopting this practice will beg no question in the debate which interests us here. So for the purposes of this paper, 'the' standard model for PA is, indifferently, $\langle\mathbb{N}, 0,1,+, \times,<\rangle$ - whatever exactly you think that is! - or any isomorphic model.

Now let $\mathcal{M}=\left\langle M, 0^{\mathcal{M}}, 1^{\mathcal{M}},+^{\mathcal{M}}, \times^{\mathcal{M}},<^{\mathcal{M}}\right\rangle$ be any model of PA, standard or nonstandard. Either the domain $M$ is countable or it isn't. In the first case, since we don't care about distinguishing isomorphic models, we can always take the domain to be $\mathbb{N}$, and then e.g. the function $+{ }^{\mathcal{M}}$ will be a function defined over the naturals. So in this case it makes sense to ask whether or not $+^{\mathcal{M}}$ is actually a recursive function. And the answer is given by the beautiful

Tennenbaum's Theorem. If $\mathcal{M}$ is a countable model of PA and $+^{\mathcal{M}}$ is recursive, then $\mathcal{M}$ is isomorphic to the standard model.

But of course, if the domain $M$ is not countable, the question whether $+^{\mathcal{M}}$ is recursive doesn't arise. So it immediately follows that the only model $\mathcal{M}$ where ${ }^{\mathcal{M}}$ is recursive is the standard model. (The same result holds for $x^{\mathcal{M}}$, but we won't need that.)

And now the following line of argument can look tempting.

\footnotetext{
${ }^{1}$ For a proof, see e.g. Kaye [1991, ch. 11].
} 
In learning arithmetic, we learn in particular how to compute addition. Reflecting on our practice, we see that genuine addition over the numbers must be a recursive function. But of course PA is intended to be, inter alia, true of genuine addition. So by Tennenbaum's Theorem, the intended model of PA just has to be the standard model.

This argument has recently gained various levels of support. To the best of our knowledge, Horsten [2010T] was the first to consider the argument, and Dean explored it with some reservations in an unpublished talk (a later version of which is available as Dean [2(1)2]). Subsequently, the argument was endorsed by both Halbach and Horsten [2005] and Quinon and Zdanowski (in an unpublished talk available as Quinon and Zdanowski [2006]).

It's not that we think this argument is unsound. On the contrary. But we don't think it that it should convince someone with sceptical worries about our grasp of the standard model.

That's obvious if the sceptic is driven by nominalist doubts about the very existence of abstract entities such as the standard model: nominalism can't be refuted by appeal to model theory! It's equally obvious if the sceptic is driven by a very general epistemological doubt that we can get any handle at all on any abstracta: if you doubt whether we can ever really know about the entities postulated in a mathematical discipline like model theory, then more mathematics isn't what you need.

The interesting case is that of Thoralf, as we will call him, a sceptic with worries about our grasp of the standard model which arise from specifically model-theoretic concerns. Thoralf isn't swayed by a global nominalism, and he is perhaps happy to allow us knowledge of some abstracta. But he is discomfited by the discovery that PA has multiple non-isomorphic models. If our practice of endorsing PA-theorems doesn't determine an interpretation, than what price now the idea of the 'right', the 'standard', interpretation? Thoralf's line of thought is familiar." But perhaps at least Thoralf's doubts, arising as they do from a specifically model-theoretic argument, can be quieted by appeal to more model theory?

Not so.

\section{Treating 'recursive' as a theoretical notion}

To show why the outlined argument from Tennenbaum's Theorem is unhelpful, consider the following argument which starts from a much easier result concerning models of PA:

Initial Segment Theorem. If $\mathcal{M}$ models PA and for every $m \in M$ there are only finitely many $n$ such that $n<^{\mathcal{M}} m$, then $\mathcal{M}$ is isomorphic to the standard model.

Now we argue along parallel lines:

In learning arithmetic, we learn in particular how to count backwards. Reflecting on our practice, we see that, under the genuine order relation over the numbers, any number has a finite number of

\footnotetext{
${ }^{2}$ Whether it is Thoralf Skolem's own line of thought is contentious (see e.g. Benacerraf 1985). Ultimately, it may be closer to the worries expressed by Hodes [1.984, §1.]. We return to these worries at the end of this paper.
} 
predecessors. But of course PA is intended to be, inter alia, true of genuine order over the numbers. So by the Initial Segment Theorem, the intended model of PA just has to be the standard model.

But Thoralf is evidently not going to be convinced by this argument. After all, the statement of the Initial Segment Theorem involves the phrase 'there are only finitely many $n$ such that ...' . But to understand this phrase, we need to have a determinate grasp on the idea of what counts as a finite cardinal number. However, that is precisely what we were trying to supply to the sceptic who thinks that the idea is open to multiple interpretations. So if Thoralf has a genuine worry about how we grasp the numbers in their standard ordering, this argument plainly can't help him.

The argument from Tennenbaum's Theorem faces essentially the same problem. The statement of the Tennenbaum's Theorem involves the phrase ' $+{ }^{\mathcal{M}}$ is recursive', and any proof of the Theorem will have to make free use of the notion of a recursive function. Thoralf will ask us to explain what 'recursive' means on our lips. Well, hand-waving a bit, we say that a total function is recursive so long as, for any inputs, a computer could determine in an arbitrary but finite number of steps what the output of the function is. So to understand even the statement of the Theorem, Thoralf needs to have a determinate grasp of the idea of an arbitrary but finite numbers of steps. But to grasp this, he needs to have a determinate grasp on the idea of what counts as a finite number. However, that is precisely what we were trying to supply to someone who thinks that the idea is open to multiple interpretations. So if Thoralf genuinely doesn't understand how we grasp the standard model, the argument from Tennenbaum's Theorem plainly can't help him.

In short, if there is a real difficulty about our grasp of the standard model which can be motivated by model-theoretic considerations, then neither argument will help, and for similar reasons.

Dean [20122] was aware of the general problem just raised for the simple argument from Tennenbaum's Theorem. However, he maintained that a variant argument can sustain the same conclusion. As he saw it, Tennenbaum's Theorem is

merely the tip of the iceberg.... If the meanings of complexity theoretic concepts were only fixed relative to [first-order definitions], they too would inherit the indeterminacy of finitude. (Dean $[2(1) 2$, p. 11])

He then went on to explain that, in every nonstandard model, every (standardly) recursive set is decidable in (what the model claims is) polynomial time. A natural thought now is that the model gets 'polynomial time' wrong too.

But inevitably, this is just more of the same. An algorithm runs in polynomial time just in case there are fixed $c, k$ such that, for any input of size $n$, the time required for a solution is bounded by $c n^{k}$. But if someone is to grasp this definition in its intended sense, where $c, k$ and $n$ are all standard numbers in $\mathbb{N}$, it is because they have already homed in on the standard model. And Thoralf is asking, precisely, how we can do that.

\footnotetext{
${ }^{3}$ Dean in fact went beyond this natural thought, and offered a more sophisticated argument based on complexity theory. We won't discuss this complication, however, as Dean has subsequently developed his own reservations concerning this argument [personal communication].
} 


\section{Treating 'recursive' as a practical notion}

Are we being unfair in treating 'recursive', 'polynomial', and so forth, as theoretical notions, up for reinterpretation? Halbach and Horsten [20(1). p. 180] want to defend the argument from Tennenbaum's Theorem, but they agree that it is 'obviously useless' to focus on theoretical recursion, 'because it singles out a certain class of functions and relations in a way that presupposes the natural numbers or set theory'. Instead, they ask us to understand recursiveness as a practical notion which concerns our ability to manipulate signs [2005, pp. 180-2]. Similarly, when Dean asked us to consider what can be decided in polynomial time, Dean had in mind not a theoretical notion, but rather 'real world limitations on our abilities' [20()2, pp. 11-12].

Defenders of the Tennenbaum argument, then, hope that we can be introduced to the notion of practical recursion, by reflecting on our real world limitations. We claim that this doesn't help matters either. Our actual limitations reveal only that we are pretty good at working out sums, products, and the like, for tractably small numbers. But if we want to talk more sweepingly, e.g. about what can or cannot be done in polynomial time (but without setting any upper limit), we need to talk about what we could do in principle. Again, by 'in principle', we mean 'given world enough, and time', i.e. given an arbitrary finite number of stages in a computation. And again, that is to assume we grasp precisely what Thoralf is challenging our entitlement to.

If this isn't clear by itself, then just observe that exactly the same moves could be offered in defence of the argument from the Initial Segment Theorem. The notion of having only finitely many predecessors can surely be given in terms of 'real world limitations': for example, if you imagine writing down the numbers using Hilbert's stroke notation, and understand 'predecessor' in terms of 'substring', we could suggest that 'finitely many' simply means 'anything which you can have written down'. More simply, we could just say that the natural numbers are those which can be so enscribed. But our actual limitations mean that we only ever succeed in writing down a few such numbers. If we want to talk about what can be written down, without upper limit, we need to talk about what we could do in principle. And by 'in principle', we mean 'given world enough, and time', i.e. given an arbitrary finite number of stages to concatenate strokes. Again, we beg the question against Thoralf.

There is a perfect parallel, then, between manœuvres which invoke Tennenbaum's Theorem and those which invoke the Initial Segment Theorem. But, sadly, those who defend the Tennenbaum argument only seem aware of the defects concerning the Initial Segment argument. Halbach and Horsten [20(15, p. 176] dislike the idea of defining the standard model in terms of canonical notations since it 'begs the question: in order to apply it, we must be able to distinguish between standard and nonstandard numerals'. True; but Halbach and Horsten require that we can distinguish between standard and nonstandard arbitrary computation procedures, which is at least as hard. Equally, Dean [20(12, p. 1] thinks that it is totally circular to define the standard numbers either as those with finitely many predecessors, or by 'iterating a concrete operation', since 'our experiences with numerals do not extend arbitrarily far along this sequence'. True; but our experiences with computations 
(and with computational complexity) are equally bounded.

The familiar point is that, just as there are divergent interpretations of the terms involved in stating Tennenbaum's Theorem (and the Initial Segment Theorem), so there are divergent interpretations of our practices of computation (and of writing down finitely many predecessors). Now, at this point an advocate of the argument from Tennenbaum's Theorem might argue that Thoralf's concerns have collapsed into a generic Kripkensteinian rule-following problem (cf. Halbach and Horsten [2005, p. 179]). This would be a mistake. Kripke [1.982, 8ff.] presents the rule-following problem thus: for suitably large $n$ and $m$, I might - consistently with all my practices to date - end up saying ' $m+n=5$ ', i.e. I might come out with a (quantifier-free) sentence which is false (false in the standard model). By constrast, in thinking about the impact of Tennenbaum's Theorem, we are considering practices which all involve endorsing PA; accordingly, there will be no deviation concerning quantifier-free sentences. Indeed, if we want, we can restrict our attention to nonstandard practices which are elementarily-equivalent to the standard practice, so that there is no deviation concerning any arithmetical sentences. All this is just to say that we are dealing with an essentially model-theoretic worry, and not just a generic rule-following problem.

\section{Philosophical problems, philosophical solutions}

To sum up the preceding two sections: if Thoralf has a genuine problem about our ability to grasp the standard model, then it is not to be tackled by appeal to Tennenbaum's Theorem. After all, that Theorem and its proof make heavy use of precisely the problematic notions. Moreover, translating the Theorem into practical terms simply reminds us that our standard mathematical notions are deeply entrenched within our practices of idealisation.

This conclusion readily generalises. If there is a genuine problem about our grasp of the standard model, then it is not to be tackled by offering any further model theoretic results either. For our model theory will extend PA precisely in order to give itself such notions as 'an arbitrary finite number', or whatever; but then Thoralf's problem about how we fix the intended interpretation of PA will just recur as a problem about how we fix the intended interpretation of the richer model theory.

For all this, we don't suppose that Thoralf's problem is a genuine problem. Indeed, in its most simple-minded form, it seems downright confused. Thoralf starts by asking us to treat all mathematical discourse as a wholly alien language, awaiting interpretation. But, to convince us that there are many possible interpretations of this language, Thoralf plays free and fast with the idea of standard and nonstandard models. In so doing, he must employ some already determinately interpreted mathematical theory, namely model theory; and that is precisely what he prohibited us all from doing at the outset! Thoralf is now mired in a dialectical tangle, and only philosophy (not more model theory) will help him out of it.

A less simple-minded Thoralf might try to avoid this muddle by claiming that, despite appearances, he is not really employing some already-interpreted model theory. Rather, he might claim, he is simply offering a reductio against his realist 
opponent, along the following lines:

Since you believe that model theory is true, you are committed (by your own lights) to the existence of many models of PA. But you can't tell a plausible story which explains how you are able to refer to one model, rather than any other. That's totally unacceptable.

This kind of challenge is dialectically more stable. And why is Thoralf so confident that no plausible story is to be had? Very likely because he thinks that

whatever the links between ourselves, our practices and abilities, and objects like Jupiter, Exxon, tyrannosaurus rex, and positrons, by virtue of which we and our words refer to them, these links are going to have to be rather different from any such links between ourselves, our practices, our abilities, and the number 1 . Numbers are so pure, so unsustained by the cement of the universe, that reference to them and their ilk seems quite sui generis.

Thoralf's challenge is well posed, but it has become extremely general. Essentially, it has the following form: since we are little more than shaved apes wearing shoes, how can we possibly manage to refer to abstract objects (numbers or otherwise)?

There's much more to be said on this question, of course, but not here. For these broad philosophical issues now have nothing specifically to do with Tennenbaum's Theorem. Suffice it to note that our discussion of Tennenbaum's Theorem illustrates a familiar moral: philosophical problems which are supposedly generated by mathematical results can rarely be tackled by offering more mathematics.

\section{Abstract}

Tennenbaum's Theorem yields an elegant characterisation of the standard model of arithmetic. Several authors have recently claimed that this result has important philosophical consequences: in particular, it offers us a way of responding to modeltheoretic worries about how we manage to grasp the standard model. We disagree. If there ever was such a problem about how we come to grasp the standard model, then Tennenbaum's Theorem doesn't help. We show this by examining a parallel argument, from a simpler model-theoretic result.

\section{References}

Benacerraf, P. (1985). "Skolem and the Skeptic". Proceedings of the Aristotelian Society, Supplementary Volumes 59, pp. 85-115.

Dean, W. (2002). "Models and Recursivity". Presented at the Pittsburgh Graduate Philosophy Conference. URL: http://citeseerx.ist.psu.edu/viewdoc/ summary?doi=10.1.1.136.446.

Halbach, V. and L. Horsten (2005). "Computational structuralism". Philosophia Mathematica 13, pp. 174-86.

Hodes, H. T. (1984). "Logicism and the Ontological Commitments of Arithmetic". The Journal of Philosophy 81.3, pp. 123-49.

\footnotetext{
${ }^{4}$ Hodes 1984, p. 127.
} 
Horsten, L. (2001). "On Our Ability to Fix Intended Structures". In Moving Ahead: Philosophy of Mind and Realism. Ed. by A. A. Derksen. Tilburg: Dutch University Press, pp. 121-34.

Kaye, R. (1991). Models of Peano Arithmetic. Oxford: Clarendon Press.

Kripke, S. A. (1982). Wittgenstein on Rules and Private Language: An elementary exposition. Cambridge MA: Harvard University Press.

Quinon, P. and K. Zdanowski (2006). "The Intended Model of Arithmetic. An Argument from Tennenbaum's Theorem". URL: http://www.impan.pl/ kz/files/ PQKZ_Tenn.pdf. 